\title{
Criteria Justification for Assessing the Technical Condition of Gearboxes of Mining Machines Based on Infrared Thermography
}

\author{
Evgeniy Kuzin ${ }^{1, *}$, Boris Gerike ${ }^{2,3}$, and Vitaliy Shachmanov ${ }^{1}$ \\ ${ }^{1}$ Branch of T.F. Gorbachev Kuzbass State Technical University in Prokopievsk, 653039, 19a \\ Nogradskaya str., Prokopievsk, Kemerovo region, Russian Federation \\ ${ }^{2}$ Federal Research Center of Coal and Coal Chemistry SB RAS, 650003, 10 Leningradsky av., \\ Kemerovo, Russian Federation \\ ${ }^{3}$ T.F. Gorbachev Kuzbass State Technical University, 650000, 28 Vesennyaya str., Kemerovo, \\ Russian Federation
}

\begin{abstract}
The paper presents the relevance of advanced assessment of the technical condition of complex technical systems. One of which is the gearbox of a mining machine. The main reasons for early failures of bearings are shown. The main share is occupied by problems of lubrication, incorrect installation and maintenance. The analysis of types of destruction of gears of mining machines is presented. The main share is occupied by the surface coloring of the teeth and sudden fracture of the tooth as a result of dynamic load. The theoretical basis for determining the heat release of bearings is given. The calculation of heat release is given of a shaft belt conveyor gearbox as an example, which is confirmed by thermographic studies under operating conditions. Justification of criteria for evaluating the technical condition of gearboxes of mining machines in the form of temperature anomalies recorded on the surface of the body, The actual load on the output shaft is taken into account.
\end{abstract}

\section{Introduction}

The issues actuality of improving the operational reliability of mining machines is proved by research of Russian and foreign authors. Thus, in [1-4] it is shown that the detection of defects in bearings and gears of mining machines is one of the most studied problems. Most of the technical systems modern components are equipped with remote monitoring systems of the machines technical conditions especially at hazardous production facilities. Effective operation of technical condition monitoring systems is achieved by creating reliable criteria for evaluating technical condition taking into the account all influencing factors. According to the authors [4 - 6], the parameters of lubricants, temperature and vibration have important diagnostic features that characterize the technical condition of most machines. Conducted research in the field of vibration diagnostics of mining machines allowed us to

\footnotetext{
* Corresponding author: kuzinevgen@gmail.com
} 
develop criteria for vibration parameters to assess the technical condition of bearings and gearboxes [7]. Another direction studying the equipment reliability is increasing the evaluation reliability of its condition at the earliest possible stage. Early detection of incipient defects allows corrective maintenance and repair due time. Using this information, you can determine the rational deadlines of repairs, predict the remaining resource of mining machine components. Moreover, and, check the quality of technical and repairing maintinence can be checked and the readiness of mining equipment can be controlled [8].

\section{Formulation of the Problem}

The calculation accuracy of the bearing life allowed to coordinate the bearing service life and that of the machine. However, the actual service life of bearings is often less than it is estimated. Among all the reasons for early failures the main ones are (Fig. 1): insufficient lubrication or inappropriate lubricants, incorrect installation and maintenance, inefficient operation of seals. The state of lubrication has the greatest impact on the bearing life and is studied in detail by bearing manufacturers $[9,10]$.

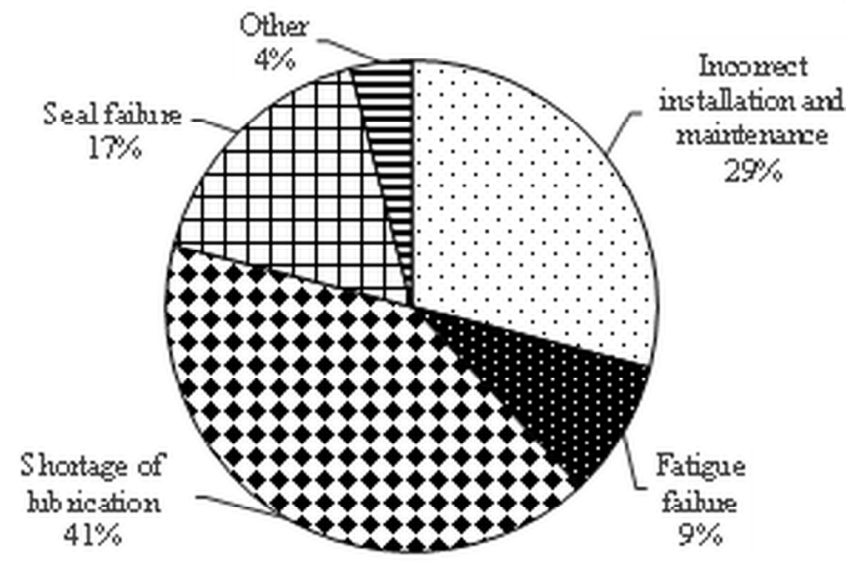

Fig. 1. Causes of rolling bearing failure.

The bearings replacement in the mining conditions is usually performed without using special tools. Installation is performed by applying a shock load, which causes the shafts to be bullied, the outer ring to be skewed relatively to the inner ring, the clearance to be exceeded when landing, or high stresses when overstretched. After disassembling the bearing it is possible to identify the cause of premature wear and take appropriate measures based on the characteristic damage pattern.

The conducted research of the destruction types of gears of gearboxes of mining machines has shown that the main destructions are (Fig. 2): discoloration of the working surfaces of the teeth; fatigue fracture of the tooth during bending; abrasive wear of the teeth; pitting of the tooth surface; sudden fracture of the tooth under the action of dynamic load (impact). 


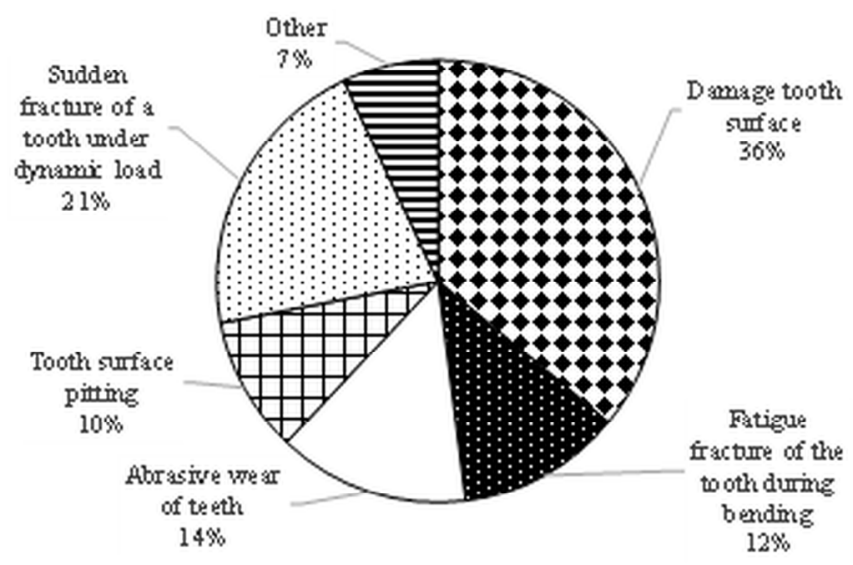

Fig. 2. Types of destruction of gears.

The most common is the staining of the working surfaces of the teeth of wheels and gears, which occurs as a result of the action of variable tenses on the working surface of the teeth, causes the appearing and developing of fatigue cracks (Fig. 3). Should be noted that the detected pitting on the surface of the teeth with further developing will lead to fatigue staining of the teeth. The speed of the staining development increases when other abrasive objects can appear in the lubricating oil.

At the same time as it is shown in the work [11], the greatest damage to the raceways can be caused by small solid particles smaller than 68 microns. During a single sample probation such particles do not always appear, and thus the spectral analysis can't define them.

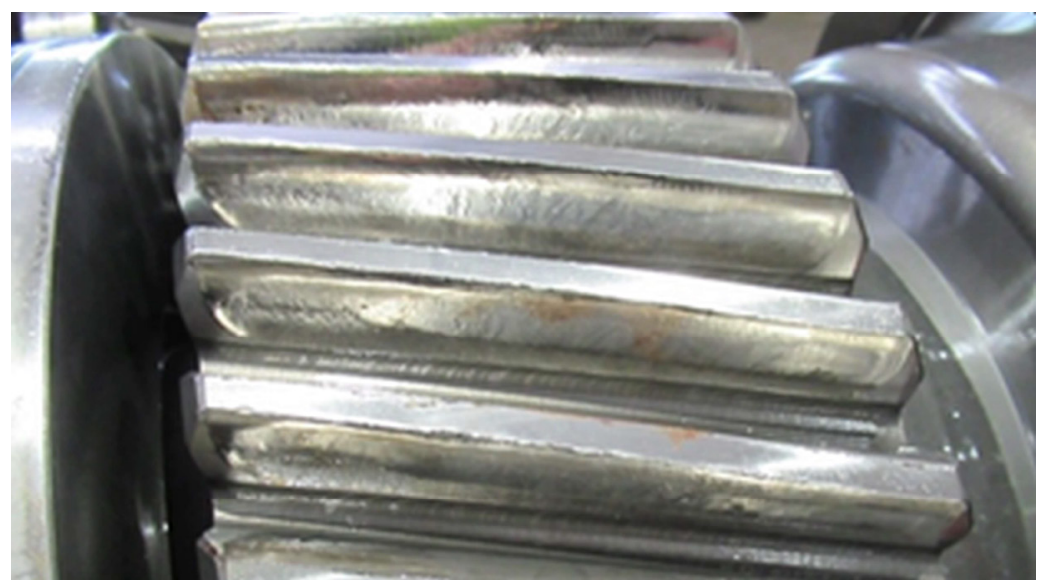

Fig. 3. Photo of destruction of working surfaces of gear teeth.

The reasons causing premature gear failures are related to contamination of the lubricating oil, the use of oil with inappropriate characteristics, shock dynamic loads, the design overloading.

It should be noted that sudden tooth breaks often occur as a secondary failure caused by complete destruction of bearings and violation of the gearing geometry.

As it practices the machine will be stopped only in the case of a planned repairing on operating time or when the vibration levels exceed the emergency levels. It can happen in fact at the last wearing stage. 
In this way, remote monitoring systems for equipment parameters are created to avoid equipment stops and early detection of wear and tear processes. A significant limitation of the diagnostic capabilities of remote monitoring systems is the lack of available criteria for evaluating the technical condition of the measured parameters set.

\section{The results of the study}

One of the ways to determine the occurrence of defects in both bearings and gears is thermal monitoring. The use of the thermal monitoring is widely known, and is successfully used for diagnostic purposes $[12,13]$.

This paper provides calculations and justification of thermal control criteria for infrared thermography of the surface of gearboxes of mine belt conveyors. The thermal balance of the generated and dissipated power in the rolling bearing (W) is determined by the method of empirical calculation [10]:

$$
\begin{gathered}
Q_{b}=Q_{c} ; Q_{b}=1.047 \cdot 10^{-4} \cdot M \cdot n ; \\
Q_{c}=28.6 \cdot V_{o} \cdot \Delta T+k_{r} \cdot A_{r} \cdot \Delta T ;
\end{gathered}
$$

where $Q_{b}$ - power generated in the bearing, W;

$Q_{c}$ - power dissipation of heat flow from the bearing, $\mathrm{W}$;

$M$ - total friction moment, $\mathrm{N} \cdot \mathrm{mm}$;

$n$ - bearing rotation speed, $\min ^{-1}$;

$V_{o}$ - volume of oil pumped through the bearing, $1 / \mathrm{min}$;

$k_{r}$ - heat transfer coefficient of the bearing seat surface, $\mathrm{W} /\left(\mathrm{mm}^{2 .{ }^{\circ}} \mathrm{C}\right)$;

$A_{r}$ - heat transfer area of the bearing landing surface $\mathrm{mm}^{2}$;

$\Delta T$ - difference between the average bearing temperature and the environment, ${ }^{\circ} \mathrm{C}$.

The friction moment for bearings of mining gearboxes is determined by the formula

$$
M=f_{0} \cdot(v \cdot n)^{0.66} \cdot d_{m}^{3} \cdot 10^{-7}+f_{1} \cdot P_{1} \cdot d_{m}
$$

where $f_{0} ; f_{1}$ - friction coefficients, respectively, depending on the viscosity of the oil and the speed and load on the bearing;

$v$ - kinematic viscosity of the oil, $\mathrm{mm}^{2} / \mathrm{s}$;

$d_{m}$ - average bearing diameter, $\mathrm{mm}$;

$P_{1}$ - total bearing load, N.

Taking the average value of the electric motor currents during the steady movement of the conveyor the friction moments and thermal capacities of the bearings were determined. The temperature difference between the bearing and the environment were determined as well. The surface temperature was measured by a thermal camera FLIR i50. The values of the temperature difference between bearings and the environment was obtained by calculation and measuring as a function of the average current of the electric motor. It is shown in the graph (Fig. 4). 


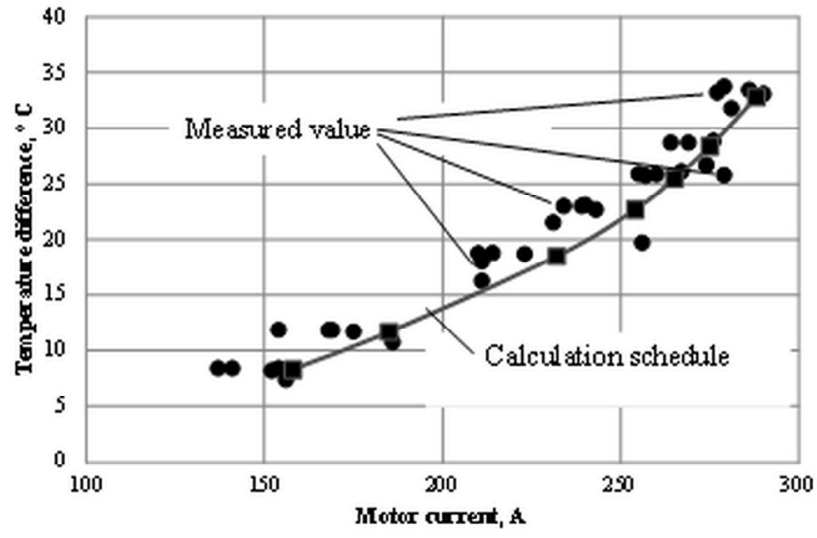

Fig. 4. Graphs of calculated and measured temperature difference between bearings and the environment of the bearing 32321A of the belt conveyor reducer.

Thermograms of Moventas D3RST82XO reducers in good technical condition a), and in the initial wear stage b) at rated load are shown in Fig. 5.
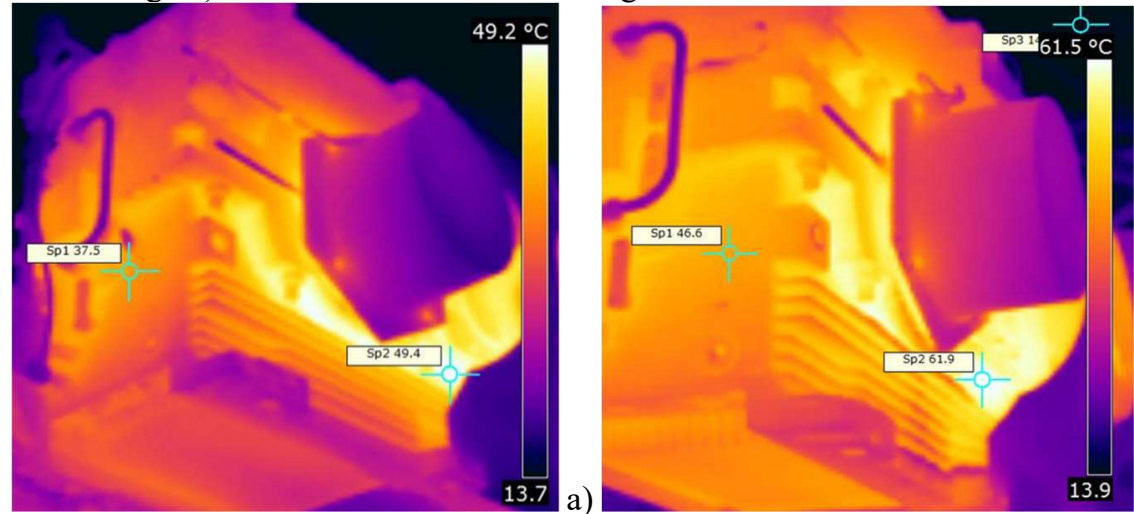

Fig. 5. Thermograms of the Moventas D3RST82XO gearbox: a) good condition, b) initial wear processes.

Analysis of the thermogram (Fig. 5, a) shows the actual excess of the temperature of the gearbox housing in the bearing area $\Delta T_{1}=49.2-15.0=34.2{ }^{\circ} \mathrm{C}$, while the current of the electric motor was $282 \mathrm{~A}$. The calculated temperature difference at this load was $\Delta T_{1}{ }^{*}=32.8^{\circ} \mathrm{C}$ and the deviation does not exceed $5 \%$.

Analysis of the thermogram (Fig. 5, b) shows the actual excess of the temperature of the gearbox housing in the bearing area $\Delta T_{2}=61.5-18.5=43.0{ }^{\circ} \mathrm{C}$, while the current of the electric motor was $288 \mathrm{~A}$. The calculated temperature difference at this load was $\Delta T_{2}{ }^{*}=33.6^{\circ} \mathrm{C}$ and the deviation exceeds $20 \%$, and was about 10 degrees.

\section{Conclusion}

The presented method for calculating heat dissipation for bearings has shown good convergence results with the measured temperatures (with averaged friction coefficients). The difference between the calculated and measured temperatures does not exceed $5.2{ }^{\circ} \mathrm{C}$.

Thus, the criterion of good technical condition of gearbox bearings should be considered when the temperature difference between the surface of the gearbox and the environment does not exceed 5 degrees. Good condition of bearings $-\Delta T \leq 5{ }^{\circ} \mathrm{C}$. 
An increase in friction coefficients of more than $15 \%$, caused by bearing wear, leads to an increase in the temperature difference between the surface of the gearbox and the environment by more than 5 degrees. The condition criterion characterizing the initial wear of bearings should be considered when the temperature difference between the surface of the gearbox and the environment is from 5 to 10 degrees $\left(5<\Delta T<10^{\circ} \mathrm{C}\right)$.

\section{References}

1. J. Obuchowski et al, Appl. Mech. Mater., 683, 171-176 (2014)

2. W. Moczulski et al, IJCRS, 9920, 33-42 (2016)

3. A. Kudrevatykh et al, E3S Web of Conferences, 105, 03021 (2019)

4. L.Jing, M. Zhao, P. Li, X. Xu, Measurement, 111, 1-10 (2017)

5. A. Karandaev, I. Yachikov, V. Khramshin, Procedia Engineering, 1, $32-38$ (2016)

6. B. Gerike, I. Panachev, E. Kuzin, E3S Web of Conferences, 15, 03008 (2017)

7. E. Kuzin, V. Shahmanov, D. Dubinkin D, E3S Web of Conferences, 21, 03006 (2017)

8. L. Andreeva, MIAB, 5, 136-143 (2018)

9. G. Morales-Espejel, Evolution, 2, 25-30 (2010)

10. Bearing damage and failure analysis, SKF Group, PUB BU/I3 14219/2 EN, 106 (2017)

11. D. Koulocheris, et al Modern Mechanical Engineering, 4, 161-170 (2013)

12. S. Sampath et al, Adv. Engg. Tech., 5, 123-128 (2015)

13. G. Meneghetti et al, Frattura ed Integrità Strutturale, 35, 172-181 (2016) 